\title{
Four prime squares and powers of 2
}

\author{
by
}

Hongze Li (Shanghai)

1. Introduction. Let

$$
\begin{aligned}
& \mathcal{A}_{3}=\{n: n \in \mathbb{N}, n \equiv 3(\bmod 24), n \not \equiv 0(\bmod 5)\}, \\
& \mathcal{A}_{5}=\{n: n \in \mathbb{N}, n \equiv 5(\bmod 24)\} .
\end{aligned}
$$

In 1938 Hua [3] proved that almost all $n \in \mathcal{A}_{3}$ are representable as sums of three squares of primes, and all sufficiently large $n \in \mathcal{A}_{5}$ are representable as sums of five squares of primes. In view of these results and Lagrange's theorem of four squares, it is reasonable to conjecture that every large even integer $n \equiv 4(\bmod 24)$ is a sum of four squares of primes

$$
n=p_{1}^{2}+p_{2}^{2}+p_{3}^{2}+p_{4}^{2} .
$$

In [6], Liu, Liu and Zhan proved that every large even integer $N$ can be written as a sum of four squares of primes and powers of 2 ,

$$
N=p_{1}^{2}+p_{2}^{2}+p_{3}^{2}+p_{4}^{2}+2^{\nu_{1}}+\cdots+2^{\nu_{k}} .
$$

In [4], Liu and Liu showed that $k=8330$ is acceptable in (1.2). Recently, Liu and Lü [7] improved this result and proved that $k=165$ suffices.

In this note, we will prove the following:

THEOREM. Every sufficiently large even integer can be written as a sum of four squares of primes and 151 powers of 2 .

Throughout this paper, $\varepsilon$ always denotes a sufficiently small positive number, though it may be different at each appearance.

2. Outline and preliminary results. Suppose $N$, our main parameter, is "sufficiently large". We write

$$
P=N^{1 / 5-\varepsilon}, \quad Q=N P^{-1} L^{14}, \quad L=\log _{2} N .
$$


The circle method, in the form we require here, begins with the observation that

$$
\begin{aligned}
R(N) & :=\sum_{\substack{N=p_{1}^{2}+\cdots+p_{4}^{2}+2^{\nu_{1}}+\cdots+2^{\nu_{k}} \\
p_{1}, \ldots, p_{4} \leq N^{1 / 2}}}\left(\log p_{1}\right) \cdots\left(\log p_{4}\right) \\
& =\int_{0}^{1} f^{4}(\alpha) g^{k}(\alpha) e(-\alpha N) d \alpha,
\end{aligned}
$$

where we write $e(x)=\exp (2 \pi i x)$ and

$$
f(\alpha)=\sum_{p^{2} \leq N}(\log p) e\left(\alpha p^{2}\right), \quad g(\alpha)=\sum_{2^{\nu} \leq N} e\left(\alpha 2^{\nu}\right):=\sum_{\nu \leq L} e\left(\alpha 2^{\nu}\right) .
$$

By Dirichlet's lemma on rational approximation, each $\alpha \in[1 / Q, 1+1 / Q]$ can be written as

$$
\alpha=\frac{a}{q}+\beta, \quad|\beta| \leq q^{-1} Q^{-1},
$$

for some integers $a, q$ with $1 \leq a \leq q \leq Q,(a, q)=1$. We denote by $I(a, q)$ the set of $\alpha$ satisfying (2.4), and define the major arcs $\mathfrak{M}$ and the minor arcs $\mathfrak{m}$ as follows:

$$
\mathfrak{M}=\bigcup_{1 \leq q \leq P} \bigcup_{\substack{a=1 \\(a, q)=1}}^{q} I(a, q), \quad \mathfrak{m}=[1 / Q, 1+1 / Q] \backslash \mathfrak{M} .
$$

It follows from $2 P<Q$ that the major arcs $I(a, q)$ are mutually disjoint.

By $(2.2)$ we have

$$
\begin{aligned}
R(N) & =\int_{\mathfrak{M}} f^{4}(\alpha) g^{k}(\alpha) e(-\alpha N) d \alpha+\int_{\mathfrak{m}} f^{4}(\alpha) g^{k}(\alpha) e(-\alpha N) d \alpha \\
& =: R_{\mathfrak{M}}(N)+R_{\mathfrak{m}}(N) .
\end{aligned}
$$

We will prove that $R(N)>0$ for sufficiently large $N$; this proves the Theorem.

For the integral on the major arcs, we need the following lemma.

Lemma 1 ([7, Lemma 2.1]). Let $\mathfrak{M}$ be as in (2.5) with $P, Q$ determined by (2.1). Then for $2 \leq n \leq N$, we have

$$
\int_{\mathfrak{M}} f^{4}(\alpha) e(-\alpha n) d \alpha=\frac{\pi^{2}}{16} \mathfrak{S}(n) n+O\left(\frac{N}{\log N}\right),
$$

where $\mathfrak{S}(n)$ is defined in $(4.4)$, and satisfies $\mathfrak{S}(n) \gg 1$ for $n \equiv 4(\bmod 24)$.

On the minor arcs, we need estimates for the measure of the set

$$
\mathcal{E}_{\lambda}:=\{\alpha \in(0,1]:|g(\alpha)| \geq \lambda L\} .
$$


The following lemma is due to Heath-Brown and Puchta [1] and calculated by Liu and Lü [7].

Lemma 2. We have meas $\left(\mathcal{E}_{\lambda}\right) \ll N^{-E(\lambda)}$, with $E(0.887167)>3 / 4+10^{-10}$.

3. Estimation of an integral. In this section we shall estimate the integral $\int_{0}^{1}|f(\alpha) g(\alpha)|^{4} d \alpha$. We have

Lemma 3. Let $f(\alpha)$ and $g(\alpha)$ be as in (2.3). Then

$$
\int_{0}^{1}|f(\alpha) g(\alpha)|^{4} d \alpha \leq c_{1} \frac{\pi^{2}}{16} N L^{4},
$$

where

$$
c_{1} \leq\left(\frac{32^{4} \cdot 101 \cdot 1.620767}{3}+\frac{8 \cdot \log ^{2} 2}{\pi^{2}}\right)(1+\varepsilon)^{9} .
$$

To show this we need

Lemma 4. For odd $q$, let $\varepsilon(q)$ be the order of 2 in the multiplicative group of integers modulo $q$. Then the series $\sum_{q=1,2 \nmid q}^{\infty} \mu^{2}(q) / q \varepsilon(q)$ is convergent, and its value $c_{2}$ satisfies $c_{2}<1.620767$.

In Lemma 4.2 of [7], one has $c_{2}<43 / 25$.

Proof of Lemma 3. By Proposition 3 in [2], we know that the conclusion of Lemma 3.1 of [7] holds for $D=N^{1 / 16-2 \varepsilon}$. By the argument in Section 3 of [7], in the proof of Lemma 2.2 of [7], we can fix $z=N^{1 / 32-\varepsilon}$, and then we can get $c_{1} \leq(1+\varepsilon)^{6} \cdot 101 \cdot 32^{4}$ in Lemma 2.2 of [7]. Following the argument of the proof of Lemma 4.1 of [7], by Lemma 4 we get the assertion of Lemma 3.

Proof of Lemma 4. For the estimation of $c_{2}$, we follow [1]. We set

$$
\begin{aligned}
m & =\prod_{e \leq x}\left(2^{e}-1\right), \\
s(x) & =\sum_{\varepsilon(d) \leq x} k(d), \quad h(n)=\sum_{d \mid n} k(d),
\end{aligned}
$$

where $k(d)$ is the multiplicative function defined by taking

$$
k\left(p^{\alpha}\right)= \begin{cases}0, & p=2 \text { or } \alpha \geq 2 \\ 1 / p, & \text { otherwise }\end{cases}
$$

Hence

$$
\begin{aligned}
s(x) & \leq \sum_{d \mid m} k(d)=h(m) \\
& =\prod_{\substack{p \mid m \\
p>2}}\left(1+\frac{1}{p}\right) \leq \prod_{\substack{p \mid m \\
p>2}}\left(1-\frac{1}{p^{2}}\right) \prod_{p \mid m} \frac{p}{p-1}=\prod_{\substack{p \mid m \\
p>2}}\left(1-\frac{1}{p^{2}}\right) \frac{m}{\phi(m)} .
\end{aligned}
$$


Moreover, we have

$$
\frac{m}{\phi(m)} \leq e^{\gamma} \log x \quad \text { for } x \geq 9,
$$

as shown in (3.9) of [5]. When $x \geq 9$ we have

$$
\prod_{\substack{p \mid m \\ p>2}}\left(1-\frac{1}{p^{2}}\right) \leq 0.831951343,
$$

hence for $x \geq 9$, we have

$$
s(x) \leq 1.4817719 \log x .
$$

It then follows that

$$
\begin{aligned}
c_{2} & =\int_{1}^{\infty} s(x) \frac{d x}{x^{2}}=\int_{1}^{9} s(x) \frac{d x}{x^{2}}+\int_{9}^{\infty} s(x) \frac{d x}{x^{2}} \\
& \leq \sum_{\varepsilon(d) \leq 9} \int_{\varepsilon(d)}^{9} k(d) \frac{d x}{x^{2}}+1.4817719 \int_{9}^{\infty} \log x \frac{d x}{x^{2}} \\
& \leq \sum_{\varepsilon(d)<9} k(d)\left(\frac{1}{\varepsilon(d)}-\frac{1}{9}\right)+1.4817719 \frac{1+\log 9}{9} .
\end{aligned}
$$

Let

$$
\sum_{\varepsilon(d)=e} k(d)=\kappa(e)
$$

Then

$$
\sum_{e \mid d} \kappa(e)=\sum_{\varepsilon(e) \mid d} k(e) .
$$

However, $\varepsilon(e) \mid d$ if and only if $e \mid 2^{d}-1$, hence

$$
\sum_{e \mid d} \kappa(e)=\sum_{e \mid 2^{d}-1} k(e)=h\left(2^{d}-1\right) .
$$

Therefore

$$
\kappa(e)=\sum_{d \mid e} \mu(e / d) h\left(2^{d}-1\right)
$$

and then

$$
\sum_{\varepsilon(d)<9} k(d)\left(\frac{1}{\varepsilon(d)}-\frac{1}{9}\right)=\sum_{m<9} \kappa(m)\left(\frac{1}{m}-\frac{1}{9}\right) .
$$

By using the information on the prime factorization of $2^{d}-1$ for $d<9$, we 
find that

$$
\sum_{m<9} \kappa(m)\left(\frac{1}{m}-\frac{1}{9}\right)=1.094371632 \ldots
$$

and hence we have

$$
c_{2} \leq \sum_{m<9} \kappa(m)\left(\frac{1}{m}-\frac{1}{9}\right)+1.4817719 \frac{1+\log 9}{9} \leq 1.6207669 \ldots
$$

This completes the proof of the lemma.

4. Proof of Theorem. For the proof, we need the following lemmas.

Lemma 5. Let $\mathcal{A}(N, k)=\left\{n \geq 2: n=N-2^{\nu_{1}}-\cdots-2^{\nu_{k}}\right\}$ with $k \geq 100$. Then for $N \equiv 4(\bmod 8)$,

$$
\sum_{\substack{n \in \mathcal{A}(N, k) \\ n \equiv 4(\bmod 24)}} n \geq\left(1 / 3-2^{-90}\right) N L^{k} .
$$

In Lemma 6.1 of [4], one has $1 / 3$ replaced by $1 / 4$.

Proof. Following the argument of Lemma 6.1 in [4], we have

$$
\sum_{\substack{n \in \mathcal{A}(N, k) \\ n \equiv 4(\bmod 24)}} n \geq \sum_{((\nu))}\left(N-2^{\nu_{1}}-\cdots-2^{\nu_{k}}\right) \geq(N-N / L) \sum_{((\nu))} 1
$$

where $((\nu))$ means $\nu_{1}, \ldots, \nu_{k}$ satisfy

$$
3 \leq \nu_{1}, \ldots, \nu_{k} \leq \log _{2}(N / k L), \quad 2^{\nu_{1}}+\cdots+2^{\nu_{k}} \equiv N-4(\bmod 3)
$$

Let

$$
H(d, N, K)=\sharp\left\{\left(\nu_{1}, \ldots, \nu_{K}\right): 1 \leq \nu_{i} \leq \varepsilon(d), d \mid N-\sum 2^{\nu_{i}}\right\} .
$$

When $d=3, \varepsilon(3)=2$, and it is an easy exercise to check that

$$
H(3, N, K)= \begin{cases}\frac{1}{3}\left(2^{K}-(-1)^{K}\right), & 3 \nmid N, \\ \frac{1}{3}\left(2^{K}+(-1)^{K}\right), & 3 \mid N .\end{cases}
$$

Thus if $K>100$ we have

$$
H(3, N, K) \varepsilon(3)^{-K} \geq \frac{1}{3}\left(1-2^{-98}\right) .
$$

And

$$
\sum_{((\nu))} 1 \geq \frac{1}{3}\left(1-2^{-98}\right) H(3, N, k)\left(\left[\log _{2}(N / k L) / \varepsilon(3)\right]-2\right)^{k} \geq \frac{1}{3}\left(1-2^{-96}\right) L^{k} .
$$

From this and (4.1) we get Lemma 5. 
Lemma 6. Let

$$
\begin{gathered}
C(q, a)=\sum_{\substack{m=1 \\
(m, q)=1}}^{q} e\left(\frac{a m^{2}}{q}\right), \quad B(n, q)=\sum_{\substack{a=1 \\
(a, q)=1}}^{q} C^{4}(q, a) e\left(-\frac{a n}{q}\right), \\
A(n, q)=\frac{B(n, q)}{\varphi^{4}(q)}, \quad \mathfrak{S}(n)=\sum_{q=1}^{\infty} A(n, q) .
\end{gathered}
$$

Then for $n \equiv 4(\bmod 24)$, one has

$$
\mathfrak{S}(n)>c_{3}
$$

with $c_{3}=4.99457$, while for $n \not \equiv 4(\bmod 24)$, one has $\mathfrak{S}(n)=0$.

In Lemma 5.2 of [7], one has $\mathfrak{S}(n)>4.952$.

Proof. This is Proposition 4.3 in [6] except for the value of $c_{3}$. It has been shown in [6] that

$$
\mathfrak{S}(n)=(1+A(n, 2)+A(n, 4)+A(n, 8)) \prod_{p \geq 3}(1+A(n, p)),
$$

where $A(n, p)$ is defined in (4.4). By the proof of Lemma 4.2 in [6], for $n \equiv 4$ $(\bmod 24)$ we have

$$
1+A(n, 2)+A(n, 4)+A(n, 8)=8, \quad 1+A(n, 3)=3 .
$$

By the proof of Lemma 5.2 in [7] we have

$$
B(n, p) \geq \begin{cases}-5 p^{2}+2 p-1 & \text { if } p \nmid n, p \equiv 3(\bmod 4), \\ -5 p^{2}-10 p-1 & \text { if } p \nmid n, p \equiv 1(\bmod 4), \\ (p-1)\left(p^{2}-6 p+1\right) & \text { if } p \mid n .\end{cases}
$$

Hence

$$
\begin{aligned}
\prod_{p \geq 5}(1+A(n, p)) \geq & \prod_{\substack{p \equiv 1(\bmod 4) \\
p \geq 5, p \nmid n}}\left(1-\frac{5 p^{2}+10 p+1}{(p-1)^{4}}\right) \\
\times & \prod_{\substack{p \equiv 3(\bmod 4) \\
p \geq 5, p \nmid n}}\left(1-\frac{5 p^{2}-2 p+1}{(p-1)^{4}}\right) \prod_{\substack{p \geq 5 \\
p \mid n}}\left(1+\frac{p^{2}-6 p+1}{(p-1)^{3}}\right) \\
> & \prod_{p \equiv 1(\bmod 4)}\left(1-\frac{5 p^{2}+10 p+1}{(p-1)^{4}}\right) \prod_{p \equiv 5(\bmod 4)}\left(1-\frac{5 p^{2}-2 p+1}{(p-1)^{4}}\right) .
\end{aligned}
$$

We apply the elementary inequality

$$
(1+x)^{a}<1+a x-\frac{a(a-1)}{2} x^{2} \quad \text { if } a>2,-1<x<0 .
$$


For $p>82$ and $p \equiv 1(\bmod 4)$, we have

$$
1-\frac{5 p^{2}+10 p+1}{(p-1)^{4}} \geq\left(1-\frac{1}{(p-1)^{2}}\right)^{5.25}
$$

and for $p>35$ and $p \equiv 3(\bmod 4)$, we have

$$
1-\frac{5 p^{2}-2 p+1}{(p-1)^{4}} \geq\left(1-\frac{1}{(p-1)^{2}}\right)^{5.25} .
$$

Thus

$$
\begin{aligned}
& \prod_{p \geq 5}(1+A(n, p)) \\
& \geq \prod_{\substack{p \equiv 1(\bmod 4) \\
5 \leq p<82}}\left(1-\frac{5 p^{2}+10 p+1}{(p-1)^{4}}\right) \prod_{\substack{p \equiv 3(\bmod 4) \\
5 \leq p<35}}\left(1-\frac{5 p^{2}-2 p+1}{(p-1)^{4}}\right) \\
& \times \prod_{p \equiv 1(\bmod 4)}\left(1-\frac{1}{(p-1)^{2}}\right)^{5.25} \prod_{\substack{p \equiv 3(\bmod 4) \\
p>35}}\left(1-\frac{1}{(p-1)^{2}}\right)^{5.25} \\
& =\prod_{\substack{p \equiv 1(\bmod 4) \\
5 \leq p<82}}\left(1-\frac{5 p^{2}+10 p+1}{(p-1)^{4}}\right) \prod_{\substack{p \equiv 3(\bmod 4) \\
5 \leq p<35}}\left(1-\frac{5 p^{2}-2 p+1}{(p-1)^{4}}\right) \\
& \times \prod_{\substack{p \equiv 1(\bmod 4) \\
5 \leq p<82}}\left(1-\frac{1}{(p-1)^{2}}\right)^{-5.25} \prod_{\substack{p \equiv 3(\bmod 4) \\
3 \leq p<35}}\left(1-\frac{1}{(p-1)^{2}}\right)^{-5.25} \\
& \times \prod_{p \geq 3}\left(1-\frac{1}{(p-1)^{2}}\right)^{5.25} \\
& \geq 1.8422 \cdot(0.6601)^{5.25}>0.208107568 \text {, }
\end{aligned}
$$

where we have used the well known result $\prod_{p \geq 3}\left(1-1 /(p-1)^{2}\right)=0.6601 \ldots$ By (4.5) and (4.6) the lemma follows.

Now we prove the Theorem. Following the argument of [7], suppose first $N \equiv 4(\bmod 8)$, let $\mathcal{E}_{\lambda}$ be defined in $(2.8)$, and $\mathfrak{M}$ and $\mathfrak{m}$ as in $(2.5)$ with $P, Q$ determined in (2.1). Then (2.2) becomes

$$
R(N)=\int_{0}^{1} f^{4}(\alpha) g^{k}(\alpha) e(-\alpha N) d \alpha=\int_{\mathfrak{M}}+\int_{\mathfrak{m} \cap \mathcal{E}_{\lambda}}+\int_{\mathfrak{m} \backslash \mathcal{E}_{\lambda}} .
$$


For the major arcs, by Lemma 1 we have

$$
\begin{aligned}
\int_{\mathfrak{M}} f^{4}(\alpha) g^{k}(\alpha) e(-\alpha N) d \alpha & =\sum_{n \in \mathcal{A}(N, k)} \int_{\mathfrak{M}} f^{4}(\alpha) e(-\alpha n) d \alpha \\
& =\frac{\pi^{2}}{16} \sum_{n \in \mathcal{A}(N, k)} \mathfrak{S}(n) n+O\left(N L^{k-1}\right) \\
& \geq c_{3} \frac{\pi^{2}}{16}\left\{\sum_{\substack{n \in \mathcal{A}(N, k) \\
n \equiv 4(\bmod 24)}} n\right\}+O\left(N L^{k-1}\right) \\
& \geq \frac{4.99457}{3}\left(1-2^{-90}\right) \frac{\pi^{2}}{16} N L^{k},
\end{aligned}
$$

where we have used Lemmas 5 and 6 .

For the second integral in (4.8), by the estimation in [7], we have

$$
\max _{\alpha \in \mathfrak{m}}|f(\alpha)| \ll N^{1 / 2-1 / 16+\varepsilon} .
$$

Therefore

$$
\int_{\mathfrak{m} \cap \mathcal{E}_{\lambda}} \ll N^{-E(0.887167)} N^{2-1 / 4+\varepsilon} L^{k} \ll N^{1-\varepsilon},
$$

where we have used Lemma 2 for $\lambda=0.887167$.

For the last integral in (4.8), by the definition of $\mathcal{E}_{\lambda}$ and Lemma 3, we have

$$
\int_{\mathfrak{m} \backslash \mathcal{E}_{\lambda}} \leq(\lambda L)^{k-4} \int_{0}^{1}|f(\alpha) g(\alpha)|^{4} d \alpha \leq c_{1} \lambda^{k-4} \frac{\pi^{2}}{16} N L^{k} .
$$

Combining this and (4.9) and (4.10), we get

$$
R(N) \geq \frac{\pi^{2}}{16} N L^{k}\left(\frac{4.99456}{3}-c_{1} \lambda^{k-4}\right) .
$$

When $k \geq 149$, for $\lambda=0.887167$, by the above estimate we have

$$
R(N)>0 .
$$

This means that every large even integer $N$ with $N \equiv 4(\bmod 24)$ can be written in the form of (1.2) for $k \geq 149$.

If $N$ is a large even integer but $N \not \equiv 4(\bmod 24)$, then by the argument of [7], $N$ can be written in the form (1.2) for $k \geq 151$. This completes the proof of the Theorem.

Acknowledgments. This work was supported by the National Natural Science Foundation of China (Grant No. 10471090). 


\section{References}

[1] D. R. Heath-Brown and J. C. Puchta, Integers represented as a sum of primes and powers of two, Asian J. Math. 6 (2002), 535-565.

[2] D. R. Heath-Brown and D. I. Tolev, Lagrange's four squares theorem with one prime and three almost-prime variables, J. Reine Angew. Math. 558 (2003), 159-224.

[3] L. K. Hua, Some results in additive prime number theory, Quart. J. Math. Oxford 9 (1938), 68-80.

[4] J. Y. Liu and M. C. Liu, Representation of even integers as sums of squares of primes and powers of 2, J. Number Theory 83 (2000), 202-225.

[5] J. Y. Liu, M. C. Liu and T. Z. Wang, On the almost Goldbach problem of Linnik, J. Théor. Nombres Bordeaux 11 (1999), 133-147.

[6] J. Y. Liu, M. C. Liu and T. Zhan, Squares of primes and powers of 2, Monatsh. Math. 128 (1999), 283-313.

[7] J. Y. Liu and G. S. Lü, Four squares of primes and 165 powers of 2, Acta Arith. 114 (2004), 55-70.

Department of Mathematics

Shanghai Jiaotong University

Shanghai 200240

People's Republic of China

E-mail: lihz@sjtu.edu.cn 